\title{
Effect of Cutaneous Leishmaniasis on Quality of Life of Patients, a Multicentric Study in Tertiary Care Hospitals in Pakistan Using DLQI
}

\author{
Najia Ahmed ${ }^{1, ~ *}$, Aymen Naeem², Bisma Zahid ${ }^{2}$, Moizza Tahir $^{3}$, Uzma Bashir ${ }^{3}$, Sameena Kausar $^{4}$, \\ Omer Farooq $^{5}$, Saira Ahmed ${ }^{6}$ \\ ${ }^{1}$ Department of Dermatology, Pakistan Naval Ship Shifa Hospital, Bahria University Medical and Dental College, Karachi, Pakistan \\ ${ }^{2}$ Department of Dermatology, Pak Emirates Military Hospital, Army Medical College, Rawalpindi, Pakistan \\ ${ }^{3}$ Department of Dermatology, Combined Military Hospital, Quetta, Pakistan \\ ${ }^{4}$ Department of Dermatology, Combined Military Hospital, Peshawer, Pakistan \\ ${ }^{5}$ Department of Ophthalmology, Pakistan Naval Ship Shifa Hospital, Bahria University Medical and Dental College, Karachi, Pakistan \\ ${ }^{6}$ Department of Radiology, Combined Military Hospital, Lahore, Pakistan
}

Email address:

docna2002@gmail.com (N. Ahmed), aymen.naeem96@gmail.com (A. Naeem), bismazahid129@gmail.com (B. Zahid),

drmt11@gmail.com (M. Tahir),4977ub@gmail.com (U. Bashir), binisami72@gmail.com (S. Kausar), ofdoc361@gmail.com (O. Farooq), seeto834@gmail.com (S. Ahmed)

${ }^{*}$ Corresponding author

\section{To cite this article:}

Najia Ahmed, Aymen Naeem, Bisma Zahid, Moizza Tahir, Uzma Bashir, Sameena Kausar, Omer Farooq, Saira Ahmed. Effect of Cutaneous Leishmaniasis on Quality of Life of Patients, a Multicentric Study in Tertiary Care Hospitals in Pakistan Using DLQI. International Journal of Clinical and Experimental Medical Sciences. Vol. 7, No. 4, 2021, pp. 103-107. doi: 10.11648/j.ijcems.20210704.16

Received: June 13, 2021; Accepted: July 19, 2021; Published: July 27, 2021

\begin{abstract}
Cutaneous Leishmaniasis (CL) is a disease caused by the parasite "Leishmania" and is endemic in more than 90 countries of the world. Despite its vast prevalence and impact on quality of life (qol), it is one of the most neglected tropical dermatological diseases. The patients face considerable mental challenges including anxiety, depression and low self-esteem, leading to a low qol. This study aims to determine the effect of CL on qol of patients suffering from it. It is a cross-sectional study that evaluated 212 patients suffering from CL who reported at Pak Emirates Military Hospital (PEMH), Rawalpindi, Combined Military Hospital (CMH), Peshawar, PNS Shifa, Karachi and CMH, Quetta from September 2018 to March 2019. For measuring the qol, Dermatology Life Quality Index (DLQI) questionnaire was filled in voluntarily. Data on demographics and characteristics of the lesions was also collected and analyzed using SPSS 22 software. DLQI scores ranged from 0 to 29 out of a total of 30 with a mean score of $14.01 \pm 7.5$. The patients were divided into five categories based on the impact the disease had on them according to the scores obtained; 74 (35\%) were those who had a large effect and only six (3\%) had no effect. "Feelings" was the most affected area (mean 2.10 \pm 0.94$)$ followed by work $(1.64 \pm 1.23)$ and sexual function had the least effect (mean 0.86 \pm 1.04 ). CL was found to have a significant effect on qol of $180(85 \%)$ of the patients. A statistically significant association was seen between type, size and duration of lesions and patient's qol. Therefore, CL significantly influences the quality of life of patients. The ulcerated type, longer duration and larger size of the lesion had greater impact on the quality of life. Preventive measures should be taken to reduce the prevalence of the disease and hence, improve the qol of people in the endemic areas.
\end{abstract}

Keywords: Cutaneous Leishmaniasis, Dermatology, Quality of Life

\section{Introduction}

Cutaneous Leishmaniasis is a disease caused by the parasite "Leishmania" transmitted by bite of Phlebotomine Sand-fly. Leishmaniasis is endemic in more than 90 countries of the world. [1] According to WHO, a total of 700,000 - 1 
million new cases of Leishmaniasis report annually throughout the world. [2] In Pakistan, it is found in all the four provinces of Pakistan; Baluchistan, Khyber Pakhtunkhuwa, Sindh and Punjab in decreasing order of prevalence. A total of 16,648 had been reported in the country for the year 2015 in WHO report 2018. [3] A study conducted in Waziristan, Pakistan in 2013-2015 showed a prevalence rate of $3.61 \%$. [4] Despite its vast prevalence and impact on quality of life, it is one of the most neglected tropical dermatological diseases. [5, 6] The main reason behind it is the low mortality rate associated with CL. The global mean age-standardized disability-adjusted life years (DALYs) lost by CL were estimated at 0.58 per 100,000 people. [7] CL has four major forms of clinical presentation; papule, nodule, nodular ulceration and plaque. It may heal spontaneously but takes a long time and leaves disfiguring scars that have a great degree of stigmatization associated with it. [8] The patients face severe mental challenges including anxiety, depression and low self-esteem, leading to a low quality of life, as seen in other disfiguring neglected tropical diseases. [9-11] currently, no such study exists in Pakistan to assess the effect on qol of these patients.

Quality of life is the life satisfaction of a person which involves physical and mental health, education, economic, social and religious aspects. DLQI is a well reputed and time tested tool for its validity and reliability to determine the effect of chronic dermatological diseases on qol. [12] Therefore, our study will be a useful contribution to study the effect of CL on qol of our population.

\section{Materials and Methods}

After approval from institutional ethical review committee, a cross-sectional analytical study was conducted at the Dermatology Department, Pak Emirates Military Hospital (PEMH) Rawalpindi, Combined Military Hospital (CMH) Peshawar, CMH Quetta and PNS Shifa, Karachi for a duration of 6 months from September 2018 to March 2019. Convenience sampling was used to collect data. The sample size was calculated using WHO sample size calculator which gave 53 patients using a prevalence of $3.61 \%$ according to a local study and 5\% margin of error. [4] Sample size $n=\left\{z^{2} x p\right.$ $(1-p)\} / \mathcal{E}^{2}$ (where, $\mathrm{z}=\mathrm{Z}$ value $(1.95$ for $95 \%$ confidence interval), $\mathrm{p}=$ prevalence in decimal form and $\varepsilon=$ margin of error percentage in decimal form) A total of 212 patients, 53 from each study centre, participated in the study. Patients who consented to participate were included in the study while those suffering from other chronic skin diseases and systemic disorders and those with diagnosed psychological disorders were excluded from the study.

Data was collected using two questionnaires; one included demographic data and clinical features of the lesions such as type, size, site, number and duration of the lesions and the other one was the Standard one-week Dermatology Life Quality Index (DLQI) questionnaire. [12] The DLQI questionnaire measures how much a skin condition has affected your quality of life in the last seven days. It contains ten questions about six domains; symptoms \& feelings, daily activities, work and school, personal relationships and treatment. Each question is scored on a four-point scale; not at all (0), a little (1), a lot (2) and very much (3). The sum of scores is to lie between zero to 30 . A higher score shows worse quality of life. According to the scores obtained on DLQI, the effect of disease on quality of life can be divided into five classes, that of no effect (DLQI score 0-1), low effect (2-5), moderate effect (6-10), large effect (11-20), and extremely large effect (21-30). [13] Significant effect comprised of moderate, large and extremely large effect. Both English and Urdu versions of the questionnaire were used for better understanding of the questions by the participants. The questionnaire was filled after obtaining written consent and all information volunteered was kept in strict confidence.

Data were analyzed using SPSS 22 software. Quantitative variables were reported as mean $\pm \mathrm{SD}$ and qualitative variables as frequency and percentages. A one-way ANOVA test was applied to describe the associations between total DLQI score and independent variables (marital status, duration, number, type, size and site of lesions).

\section{Results}

Mean age of the patients was 29.09 $\pm 6.99 .66(31 \%)$ of the patients were unmarried and $146(69 \%)$ were married. 163 (77\%) patients had an educational status of Grade 10 or less.

The most common type of lesion was nodulo-ulcerative 92 (43.5\%) followed by plaque $56(26.5 \%)$, ulcer $50(23.5 \%)$ and papule 14 (6.5\%). Majority had a single lesion $101(48 \%)$ while $53(25 \%)$ had more than three lesions. The lesions were most commonly located on the upper limb 118 (44.9\%) followed by lower limb 106 (40.5\%), face $22(8.5 \%)$ and trunk $16(6.1 \%)$. The duration of lesions ranged from three days to three years with a mean of $3.38 \pm 4.21$ months.

DLQI scores ranged from 0 to 29 out of a total of 30 with a mean score of $14.01 \pm 7.5$. On the basis of scores obtained on DLQI, the effect of disease on quality of life was classified into five categories and the results were as presented in Figure 1.

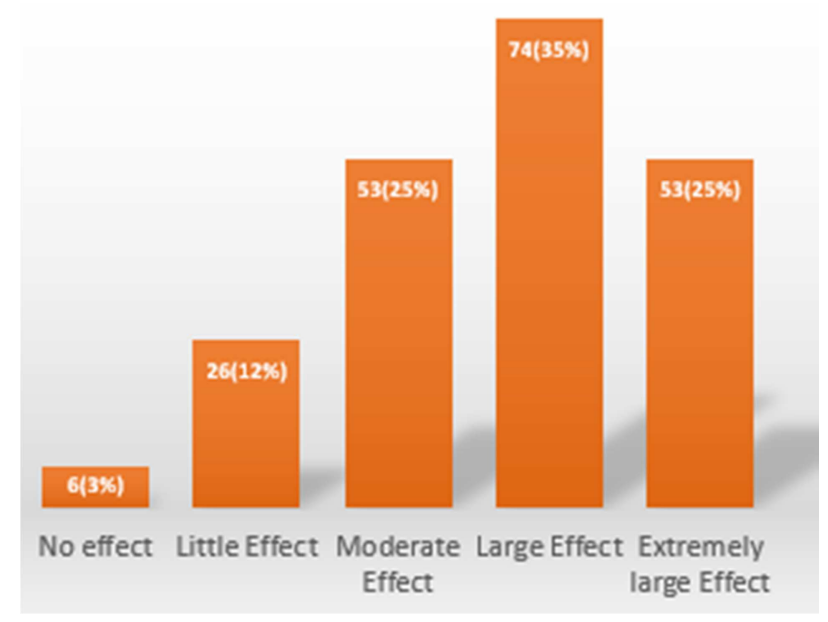

Figure 1. Effect of Cutaneous Leishmaniasis on quality of life. 
CL was found to have a significant effect on qol of 180 $(85 \%)$ of the patients. "Feelings" was the most affected area with a mean score of $2.10 \pm 0.94$ followed by work
$(1.64 \pm 1.23)$. Lowest effect was observed on sexual function $(0.86 \pm 1.04)$ as presented in Figure 2 .

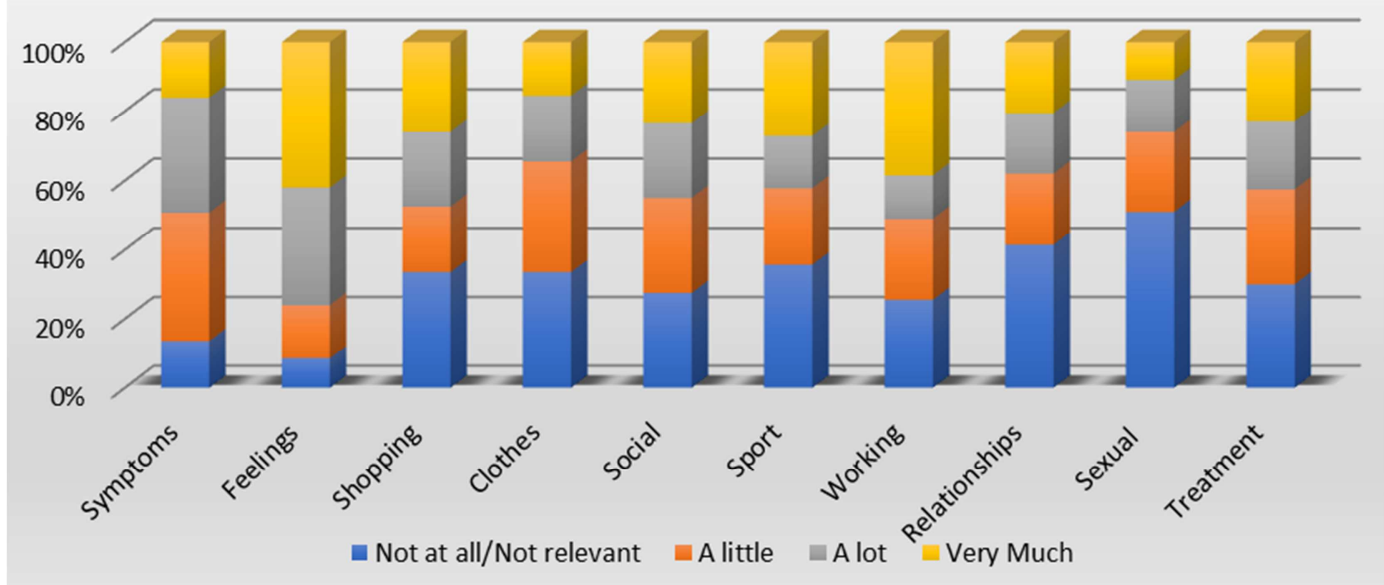

Figure 2. The proportions of affected persons in each question first to tenth using the classification used in grading each area.

A statistically significant association was found between mean DLQI scores, type, size and duration of lesions as depicted in Table 1. Patients with ulcerated lesions had a higher DLQI score indicating large effect on quality of life. Quality of life in people with papular lesions was better than in those with nodular lesions and plaques. Those with larger lesions had higher effect on their quality of life compared to those with smaller lesion. Patients with lesions more than six months were more affected than other groups. There was no significant difference between patients with respect to their marital status, educational status, and location of the lesions. However, patients with more than three lesions had a greater negative impact on their quality of life.

Table 1. Relationship of DLQI scores with Characteristics of lesions.

\begin{tabular}{|c|c|c|c|c|}
\hline Variables & Frequency & Mean & ANOVA F & Pvalue \\
\hline Type of Lesion & & & \multirow{5}{*}{3.711} & \multirow{5}{*}{0.013} \\
\hline Nodulo-ulcerative & 92 & $12.55 \pm 7.072$ & & \\
\hline Papule & 14 & $10.92 \pm 8.057$ & & \\
\hline Plaque & 56 & $15.30 \pm 6.999$ & & \\
\hline Ulcer & 50 & $16.11 \pm 8.082$ & & \\
\hline Size of lesion & & & \multirow{5}{*}{12.095} & \multirow{4}{*}{0.000011} \\
\hline$<10 \mathrm{~mm}$ & 52 & $9.84 \pm 5.662$ & & \\
\hline $10 \mathrm{~mm}-25 \mathrm{~mm}$ & 78 & $14.52 \pm 7.050$ & & \\
\hline$>25 \mathrm{~mm}$ & 82 & $16.16 \pm 7.980$ & & \\
\hline No of lesion & & & & \\
\hline 1 & 101 & $12.99 \pm 7.3$ & \multirow{3}{*}{2.705} & \multirow{3}{*}{0.069} \\
\hline $2-3$ & 58 & $13.93 \pm 8.2$ & & \\
\hline$>3$ & 53 & $16.02 \pm 6.5$ & & \\
\hline \multicolumn{5}{|l|}{ Duration of Lesion } \\
\hline$<6$ months & 182 & $13.46 \pm 7.3$ & \multirow{3}{*}{3.750} & \multirow{3}{*}{0.025} \\
\hline $6 \mathrm{~m}$ to $1 \mathrm{yr}$ & 20 & $18.21 \pm 6.0$ & & \\
\hline$>1 \mathrm{yr}$ & 10 & $15.67 \pm 10.6$ & & \\
\hline
\end{tabular}

\section{Discussion}

Currently, there are only a few studies on quality of life in patients of CL around the world. No such study has been done in Pakistan. The mean DLQI score in our study was $14.01 \pm 7.5$ indicating large effect on the quality of life. This is consistent with the findings of a study conducted in Iran that showed mean DLQI scores of 13.7 77.8. [14] Another Iranian study showed impairment of quality of life in CL patients with mean DLQI of $11.7 \pm 7.5$. [15] Similar to our study, $60 \%$ of these patients had scores $>11$ with $42.5 \%$ falling in the category of largely affected, and $17.5 \%$ in the category of very severely affected based on DLQI scores as compared to $35 \%$ and $25 \%$ in ours. In contrast a study conducted in Sri Lanka evaluating the quality of life in civilians and army personnel with CL found it to be negligibly affected by the disease overall. [16]

The characteristics of the lesions were similar to those in a research carried out in Sibbi, Baluchistan with ulcerative lesions being the most frequent clinical presentation followed by plaques, and with most lesions located on the upper limb followed by lower limb. [17] We found a statistically significant association between mean DLQI scores and type, duration and size of lesions. Ulcerative lesions caused the most disfigurement and hence the patients had the lowest 
quality of life. Papular lesions had higher qoL probably because of their smaller size and earlier appearance in the course of the disease as also demonstrated in other studies. $[15,18]$ Quality of life was the most affected in patients with lesions persisting beyond six months in our study. Evaluation of quality of life in other chronic skin disorders in a study carried out in Pakistan also showed higher DLQI scores with increasing duration of disease. [19] Noorpisheh's study also revealed direct relation of quality of life with duration of CL however it did not depict a significant effect of the size of lesions on quality of life. [14] Our results indicated that larger lesions were found to have a greater negative impact on quality of life than smaller ones concordant with another Sri Lankan study done in 2014. [20]

According to DLQI, CL had the maximum influence on the area of feelings in our study while sexual function was the least affected. However they suffered low self-esteem in their interactions with their spouses due to disfiguring nature of their lesions. This is in agreement with other similar studies. $[16,18,20]$ An Indian study on Post Kalazar Dermal Leishmaniasis also demonstrated highest effect in the feelings domain and lowest in personal relationship domain. [21] This shows that although different studies have been performed in different cultural setups, affect domains are constant for all CL patients. Increased prevalence of anxiety and depression was found in a study conducted on a group of pediatric patients highlighting the psychological impact of the disease. [22] Other studies on the effect of CL on mental health show that cosmetic disfigurement caused by $\mathrm{CL}$ lesions lead to deleterious effects on self-confidence and selfbody image perception. Mood disorders are reported as a noticeable psychological problem in such patients. [23]

A finding unique to our study was that work domain was the second most commonly affected. This could be attributed to absenteeism from work in peripheral areas to seek treatment at a tertiary care setting as lesions progress. Missing work is associated with higher impact of CL on quality of life in patients along with other factors such as presence of comorbidities. [24] We excluded the patients with systemic disorders to minimize this effect. A pilot study in Brazil also found the highest effect on the work and school domain. [25]

This study had three limitation. First, it was conducted over only six months and the variation in results with time was not taken into consideration. Second, our research considered the effects on people going to endemic areas due to their job requirements not the local population living in the endemic regions. Third, there was no method to grade the severity of the lesions, only the types were taken into consideration.

\section{Conclusion}

Cutaneous Leishmaniasis significantly influences the quality of life of patients suffering from it. The ulcerated type, longer duration and larger size of the lesion had greater impact on the quality of life. "Feelings" was the most affected domain followed by work, whereas sexual function was the least affected.

More studies with larger sample size and duration should be conducted to further elaborate the effect of CL on qol of the patients. Some preventive measures need to be introduced to reduce the prevalence of Leishmaniasis such as the use of insecticide sprays and fly nets. This aims to improve the quality of life of people in the endemic area and lower the incidence of anxiety, depression and low self-esteem.

\section{References}

[1] Rasheed Z, Ahmed AA, Salem T, Al-Dhubaibi MS, Al Robaee AA, Alzolibani AA. Prevalence of Leishmania species among patients with cutaneous leishmaniasis in Qassim province of Saudi Arabia. BMC public health. 2019; 19 (1): 384. Doi: https://doi.org/10.1515/jtim-2015-0002.

[2] Who. int. $2020 \mathrm{https} / / /$ www.who.int/news-room/factsheets/detail/leishmaniasis.

[3] Who. int. 2018 https://www.who.int/leishmaniasis/burden/Pakistan_2015.pdf? ua $=1$.

[4] Hussain M, Munir S, Khan TA, Khan A, Ayaz S, Jamal MA, et al. Epidemiology of cutaneous leishmaniasis outbreak, Waziristan, Pakistan. Emerging infectious diseases. 2018; 24 (1): 159. Doi: https://doi.org/10.3201/eid2401.170358.

[5] Molyneux DH, Savioli L, Engels D. Neglected tropical diseases: progress towards addressing the chronic pandemic. The Lancet. 2017; 389 (10066): 312-25. Doi: https://doi.org/10.1016/S0140-6736 (16)30171-4.

[6] Mokni M. Leishmanioses cutanées [Cutaneous leishmaniasis]. Ann Dermatol Venereol. 2019; 146 (3): 232-246. French. doi: 10.1016/j.annder.2019.02.002.

[7] Karimkhani C, Wanga V, Coffeng LE, Naghavi P, Dellavalle RP, Naghavi M. Global burden of cutaneous leishmaniasis: a cross-sectional analysis from the Global Burden of Disease Study 2013. The Lancet Infectious Diseases. 2016; 16 (5): 584-91. Doi: https://doi.org/10.1016/S1473-3099 (16)00003-7.

[8] Bailey F, Mondragon-Shem K, Haines LR, Olabi A, Alorfi A, Ruiz-Postigo JA et al. Cutaneous leishmaniasis and co-morbid major depressive disorder: A systematic review with burden estimates. PLoS neglected tropical diseases. 2019; 13 (2): e0007092. Doi: https://doi.org/10.1371/journal.pntd.0007092.

[9] Gurel MS, Tekin B, Uzun S. Cutaneous leishmaniasis: A great imitator. Clin Dermatol. 2020; 38 (2): 140-151. doi: 10.1016/j.clindermatol.2019.10.008.

[10] Bennis I, De Brouwere V, Belrhiti Z, Sahibi H, Boelaert M. Psychosocial burden of localised cutaneous Leishmaniasis: a scoping review. BMC Public Health. 2018; 18 (1): 358. Doi: https://doi.org/10.1186/s12889-018-5260-9.

[11] Chahed MK, Bellali H, Jemaa SB, Bellaj T. Psychological and psychosocial consequences of zoonotic cutaneous leishmaniasis among women in Tunisia: preliminary findings from an exploratory study. PLoS neglected tropical diseases. 2016 Oct; $\quad 10 \quad$ (10). https://doi.org/10.1371/journal.pntd.0005090. 
[12] Finlay AY, Khan GK. Dermatology Life Quality Index (DLQI) A simple practical measure for routine clinical use. Clinical and Experimental Dermatology. 1994; 19: 210-216.3 Doi: https://doi.org/10.1111/j.1365-2230.1994.tb01167.x.

[13] Hongbo Y, Thomas CL, Harrison MA, Salek MS, Finlay AY Translating the science of quality of life into practice: What do Dermatology Life Quality Index score mean? Journal of Investigative Dermatology. 2005; 125: 659-664. Doi: https://doi.org/10.1111/j.0022-202X.2005.23621.x.

[14] Noorpisheh S, Naghizadeh MM, Nikrouz L. A study on the life quality of patients suffering from leishmaniasis. Journal of Fasa University of Medical Sciences. 2013; 3 (2): 155-162.

[15] Khoshnood B, Handjani F, Goodarzi H, Sadati MS. Quality of life in patients with cutaneous leishmaniasis. Hormozgan Medical Journal. 2016; 20 (5): 332-336.

[16] Refai WF, Madarasingha NP, Sumanasena B, Weerasingha S, Fernandopulle R, Karunaweera ND. Cutaneous leishmaniasis in Sri Lanka: effect on quality of life. International journal of dermatology. 2018; 57 (12): 1442-1446. Doi: https://doi.org/10.1111/ijd.14240.

[17] Ahmad I, e Humayun Z, Ahmad M. Pattern of cutaneous leishmaniasis cases among troops and their families in Sibi. Pakistan Armed Forces Medical Journal. 2008; 58 (2): 209212.

[18] Vares B, Mohseni M, Heshmatkhah A, Farjzadeh S, ShamsiMeymandi S, Rahnama Z et al. Quality of life in patients with cutaneous leishmaniasis. Archives of Iranian medicine. 2013 Aug 1; 16 (8): 474.

[19] Ejaz A, Rao SE, Manzoor A, Niaz A. Quality of life assessment in chronic skin disorders. Journal of Pakistan Association of Dermatology. 2016; 25 (2): 86-89.
[20] Ranawaka RR, Weerakoon HS, de Silva SH. The quality of life of Sri Lankan patients with cutaneous leishmaniasis. Mymensingh Med J. 2014; 23 (2): 345-51.

[21] Pal B, Murti K, Siddiqui NA, Das P, Lal CS, Babu R et al. Assessment of quality of life in patients with post kalaazar dermal leishmaniasis. Health and quality of life outcomes. 2017; 15 (1): 148. Doi: https://doi.org/10.1186/s12955-0170720-y.

[22] Shiva F, Seifi N, Yaghoubi M, Momenzadeh A, Moradi A, Darchini-Maragheh E et al. Evaluation of Quality of Life, Anxiety and Depression in Children with Cutaneous Leishmaniasis. International Journal of Pediatrics. 2019; 7 (6): 9591-9600.

Doi: https://dx.doi.org/10.22038/IJP.2019.38495.3301.

[23] Bennis I, Thys S, Filali H, Brouwere V, Sahibi H, Boelaert M. Psychosocial impact of scars due to cutaneous leishmaniasis on high school students in Errachidia province, Morocco. Infect Dis Poverty. 2017; 6 (1): 46. Doi: https://doi.org/10.1186/s40249-017-0267-5.

[24] Galvão EL, Pedras MJ, Cota GF, Rabello A, Simões TC. How cutaneous leishmaniasis and treatment impacts in the patients' lives: A cross-sectional study. PloS one. 2019; 14 (1): e0211374. Doi: https://doi.org/10.1371/journal.pone.0211374.

[25] Toledo Jr AC, da Silva RE, Carmo RF, Amaral TA, Luz ZM, Rabello A. Assessment of the quality of life of patients with cutaneous leishmaniasis in Belo Horizonte, Brazil, 2009-2010. A pilot study. Transactions of the Royal Society of Tropical Medicine and Hygiene. 2013; 107 (5): 335-336. Doi: https://doi.org/10.1093/trstmh/trt021. 